\title{
Development of an online learning environment for geography and geology using Minecraft
}

\author{
Junko Iwahashi ${ }^{\text {a, }}$, Yoshiharu Nishioka ${ }^{b}$, Daisaku Kawabata ${ }^{b}$, Akinobu Ando ${ }^{c}$, Hiroshi Une ${ }^{\mathrm{d}}$ \\ ${ }^{a}$ Geospatial Information Authority of Japan, iwahashi-j96pz@mlit.go.jp \\ ${ }^{b}$ Geological Survey of Japan, AIST,y-nishioka@aist.go.jp, d-kawabata@aist.go.jp \\ ${ }^{c}$ Miyagi University of Education, andy@staff.miyakyo-u.ac.jp \\ ${ }^{d}$ Geospatial Information Authority of Japan (former affiliation), hiroune@mb.infoweb.ne.jp \\ * Corresponding author
}

Keywords: Minecraft, Computer game, Education, Geography, Geology, Sendai

Abstract:

The purpose of this research is to give children a geographical viewpoint, and to encourage an interest in, and awareness of, landforms and geology. We created a system based on an exploration type computer game and verified the educational effects. Moreover, we aim to reach not only the virtual aspect but we also have a goal of creating interest in the actual field. As a secondary effect, by using a computer game that attracts children's interest, we aim to make the experience of solving issues subjective and active even if the player is a passive child, a child with little inquiry, or a child who is not adept at self-assertion. With this new approach, we also hope to interact with young generations who usually do not interact with researchers.

Many thematic maps of geography and geology are already published on the Web. They are popular among those who need to collect and view the information for some reason or with those who are interested in observing topographic maps and are interested in geology. However, in particular, the approach to children who do not have such motivation needs one more step: a mechanism to induce an inquiring mind, and a mechanism that leads to finding the information and having interest in the real field.

The platform of this research is Minecraft Education Edition (Mojang/Microsoft). Minecraft is very popular game software which has exceeded one hundred million users worldwide in recent years, and in Japan there are many elementary and junior high school student enthusiasts of Minecraft. In the game a user explores a virtual world made of cubic blocks. The blocks imitate vegetation, rock formations, and other items, and can create various puzzles. In recent years, the release of the Education Edition assumes use in classrooms.

In this research, we have constructed a virtual world tailored to a specific junior high school which teaches science classes to first grade students. First, we re-created the actual school buildings and also included the underground geologic strata based on data from boring. In addition, we created a mechanism to expand children's imagination and knowledge about past environments which can be understood from the geological strata. We also provided checkpoints and gave challenges regarding knowledge about the formation of the land. Together with this modern world, we created ancient virtual worlds so users may understand the geological history around the school's location.

Through the experience of this research, we were able to confirm the mechanisms for promoting motivation in children and aiding their understanding of science. It can be applied to systems other than Minecraft, and it can contribute to educational support in a wide variety of fields.

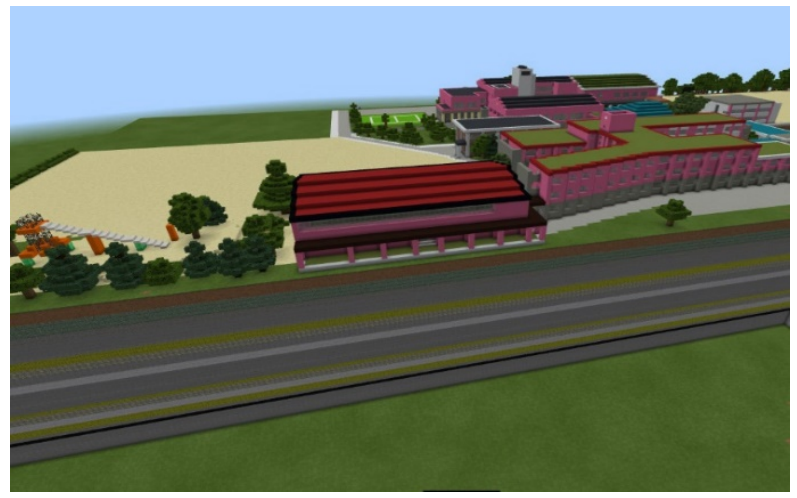

Figure 1. Overview of the school buildings and the underground geological strata.

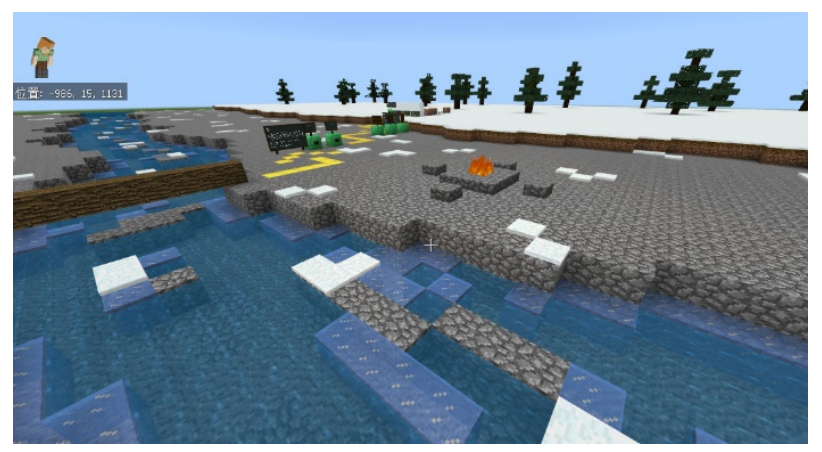

Figure 2. The appearance of the ancient world based on the geological history around the school, with path trails and questions on blackboards. 